\title{
DOCENCIA EN MEDIO DE LA PANDEMIA, UN ANÁLISIS DE LA EDUCACIÓN EN CIENCIAS DE LA SALUD
}

Ottoniel Sánchez Murgas ${ }^{1}$

Tras la Declaración de Pandemia de la COVID-19 por la Organización Mundial de la Salud (OMS) en marzo de $2020^{(1)}$ se ha obligado al personal docente a adaptarse y ver con una nueva perspectiva el proceso de formación en las Facultades de Medicina, optando por varias alternativas virtuales en los procesos de enseñanza.

Todos los niveles educativos han sido afectados durante la pandemia COVID-19, cancelando programas de intercambio y viajes al extranjero para estudiantes, docentes e investigadores. También, las reuniones académicas, los congresos internacionales y nacionales de asociaciones estudiantiles fueron afectadas; pero, sin duda, el mayor impacto lo sufrió la academia debido a la suspensión de actividades de docencia presenciales.

En medio de la cuarentena ${ }^{(2)}$, la única garantía de continuidad académica ha sido el uso de la virtualidad a través de la cual los estudiantes han debido aprender a distancia, y el docente ha debido utilizar muchas herramientas para aliviar los efectos negativos que la pandemia tiene en la educación médica, como el retraso práctico en la enseñanza de las ciencias básicas y las prácticas clínicas; aunque la práctica ha continuado durante los últimos años en las carreras en ciencias de la salud, internos, servicio social y especialidades médicas, el proceso de enseñanza-aprendizaje y la relación médico-paciente han sido afectadas. Tampoco hay acceso a las aulas, laboratorios, clínicas, tutorías, prácticas hospitalarias de externado, visitas a comunidades para medicina comunitaria, donde las escuelas hospitalarias; todas las unidades comunitarias de salud familiar de primer nivel, los hospitales de segundo y tercer nivel tienen como prioridad, en este momento, el COVID-19.

Actualmente, la virtualidad es el único escenario seguro- con todos los retos que conlleva y asumiendo la imposibilidad de reproducir en su totalidad la experiencia clínica directa- como un pilar fundamental en formación de los estudiantes de ciencias de la salud. Históricamente los estudiantes de Medicina, Enfermería y Nutrición siempre han brindado apoyo al sistema de salud ${ }^{(3)}$. Sin embargo, por la pandemia se ha obligado a suspender las actividades hospitalarias, en casi todos niveles de carreras médicas, debido a las características de alto contagio de esta enfermedad, por lo que estudiantes como el resto de la población deben protegerse. Un contraste completamente diferente ocurre a los estudiantes que cursan los últimos años de carreras de medicina, nutrición y enfermería y los residentes de especialidades médicas que han seguido trabajando en medio de esta crisis, con mucha preocupación cuando se trata de seguridad, cuidado y protección por la existencia de un

1/ Doctor en Medicina y Máster en Salud Familiar, decano de la Facultad de Medicina Universidad Evangélica de El Salvador, ottonielsanchez@gmail.com ORCID 0000-0002-7319-733X 
escenario que mezcla la responsabilidad académica y la responsabilidad laboral de los estudiantes contratados a tiempo completo por el Ministerio de Salud ${ }^{(4)}$.

A diferencia de otras disciplinas, los docentes y los estudiantes de ciencias médicas y de salud desempeñan un papel activo en el proceso de enseñanza-aprendizaje de la profesión. Pero hoy es el momento de repensar el papel que desempeñan los profesionales de la salud en formación, no solo para mantener la continuidad de sus procesos de aprendizaje sino para adaptarse y convertirse en un agente que forma parte de la respuesta sanitaria arriesgando su vida en la pandemia.

Es hora de agradecer el esfuerzo valioso de cada uno de los docentes que se han adaptado a esta nueva y difícil dinámica educativa poco explorada, es un compromiso invaluable la necesidad de continuar la formación de profesionales sanitarios con otras modalidades educativas que protejan la integridad y la seguridad, además de adaptarse a la acción de educar y salvar vidas ${ }^{(5)}$, hacer frente como trabajadores de la sTalud en medio de la pandemia.

Asimismo, las demandas de trabajo de los docentes han aumentado, además, de la adaptación rápida a la interacción virtual. Estas demandas de los estudiantes muestran las diferencias entre los docentes que conocen y dominan herramientas virtuales o los métodos de aprendizaje a distancia e incluso las condiciones familiares que afectan el trabajo de enseñanza. Casi nadie reconoce que la enseñanza en línea demanda una planificación previa de 6 meses aproximadamente, debido a que el aprendizaje basado en la interacción- y no en la transmisión de información- requiere seleccionar contenido, material propuesto y planificar el tipo de interacción que los estudiantes tendrán entre sí.

La virtualidad es considerada la gran solución a la necesidad de quedarse en casa para reducir el número de contagios. Sin embargo, a través de esta se han manifestado las desigualdades en el acceso a Internet y los recursos tecnológicos, también es evidente cuando el docente debe ser flexible ante cada uno de los casos y dificultades que presenta el estudiante. Por lo cual es necesario dar las gracias a nuestros docentes siempre listos para transmitir y facilitar el conocimiento, demostrando resiliencia para cambiar su práctica docente en beneficio de los alumnos que deben ser comprendidos y escuchados en esta crisis mundial. Hoy más que nunca los docentes se convierten en ese faro de luz que guía en estos tiempos difíciles. 


\section{Bibliografía}

1. La OMS caracteriza a COVID-19 como una pandemia - OPS/OMS | Organización Panamericana de la Salud [Internet]. [citado 14 de junio de 2020]. Disponible en: http://www.paho.org/es/noticias/113-2020-oms-caracteriza-covid-19-como-pandemia

2. El Gobierno anuncia 15 días de prórroga de la cuarentena nacional de viviendas por COVID-19 [Internet]. Presidencia de la República de El Salvador. 2020 [citado el 14 de junio de 2020]. Disponible en: https://www.presidencia.gob.sv/gobierno-anuncia-extension-de-15-dias-de-la-cuarentena-domiciliar-nacional-por-covid-19/

3. El papel del estudiante de ciencias médicas y de salud frente a una pandemia - Observatorio de la Innovación Educativa [Internet]. [citado el 12 de junio de 2020]. Disponible en: https://observatorio. tec.mx/edu-bits-blog/rol-del-estudiante-de-medicina-y-ciencias-de-la-salud-ante-una-pandemia

4. El Gobierno llega a un acuerdo con la Plaza para Todos los Movimientos (Internet). Ministerio de Trabajo y Bienestar Social de El Salvador. [citado el 14 de junio de 2020]. Disponible en: http://www.mtps. gob.sv/noticias/gobierno-logra-acuerdo-movimiento-plaza-todos-2/

5. Departamento de Computación de la Facultad de Medicina U. Prensa Artículos Revista de Investigación en Educación Médica [Internet]. Ministerio de Educación Médica. Facultad de Medicina, Universidad Nacional Autónoma de México; 2012 [citado el 21 de junio de 2020]. Disponible en: http:// riem.facmed.unam.mx/node/1080?fbclid=IwAR2bZhlKG0ozsdRQwBdv_mKfu4UDmbouCsAcCpQmARqESzEUwUPerjppPE4\# 